\title{
Accreditation of residency training in the US
}

\author{
Judith S Armbruster
}

\begin{abstract}
Summary
In the US, accreditation and certification of residency training are functions of separate public sector agencies. Accrediting decisions are made directly by 26 Residency Review Committees, which represent the primary medical specialties and function under the authority of the Accreditation Council for Graduate Medical Education. The accrediting bodies may consider only educational issues and are prohibited by the government from controlling physician supply. Only the programme, not the institution in which it is conducted, is accredited. The US residency is a structured educational programme that is expected to provide comparable experience to all enrolled residents. Length of training may vary from two to six years depending on the specialty. Additional training may be obtained in subspecialty programmes, which are subsets of the primary specialty residencies and are also reviewed for accreditation. These have increased in significant number in recent years as subspecialisation has proliferated in the US.
\end{abstract}

Keywords: US residency training, education

ACGME, Suite 2000, 515 N State Street, Chicago, IL 60610, USA JS Armbruster
In the US, graduate medical education refers to the specialised training that is taken in a residency programme following completion of medical school. Physicians who are in such programmes are referred to as residents. Responsibility for the quality of residency training is vested in two agencies. The Accreditation Council for Graduate Medical Education establishes the standards and reviews and accredits the residency programmes, while the American Board of Medical Specialties is the coordinating organisation for the specialty boards, which have as their primary function the evaluation of graduates of residency programmes to determine that they have received adequate preparation in accordance with established educational standards. While the focus of this article is on the accreditation system for residency programmes, it is necessary to first distinguish that role from the one performed by the American Board of Medical Specialties and its member boards.

\section{The American Board of Medical Specialties}

A major role played by the American Board of Medical Specialties is the acknowledgment of an area of medical practice and its designation as an officially recognised specialty. This occurs when the practice area has become sufficiently differentiated and developed to petition for its own certifying board. This step may be taken when it can be shown that a large number of physicians are engaged exclusively in the specialty, that there is a specialty society, and that there exists a body of research and scholarly publication. The American Board of Medical Specialties has now recognised 26 specialty boards, the most recent being medical genetics, which was recognized in 1991. This is the first new specialty board approved since the recognition of emergency medicine in 1979 .

As part of a proposal for membership of the American Board of Medical Specialties, it must be demonstrated that the specialty has developed a rigorous certifying examination. Although certification is not an absolute requirement, a majority of a residency programme's graduates are expected to present for the examinations, which are given by most specialties at least once a year. 'Board certified' is generally regarded by the public and by hospital credentialling committees as an imprimatur of physician quality and it is important when physicians seek clinical privileges or membership in professional societies.

\section{American Council for Graduate Medical Education}

In the US, the establishment of standards for graduate medical education by a professional organistion dates from 1914, when the American Medical Association published the first list of hospitals approved for internships. The first specialties to develop and publish standards for graduate medical education programmes were surgery and internal medicine which, in 1949, formed committees to monitor the quality of training. By the $1950 \mathrm{~s}$, a system of multiple review committees was developing as new specialties became defined. In the early 1970 s, the need to bring the different review committees under a single coordinating group was evident. The process began to assume its present configuration in which the review committees function under the aegis of an umbrella organisation that has developed a coherent programme of policies, bodies and standards for graduate medical education.

Since 1981, this body has been the American Council for Graduate Medical Education. It has responsibility for overseeing the accreditation of the residency training programmes in the 26 medical specialties recognised by the American Board of Medical Specialties, and the related subspecialties. Five major medical organisations sponsor the American Council for Graduate Medical Education and each of them is involved significantly in the training of physicians and the practice of medicine: the Association of American Medical Colleges, the American Board of Medical Specialties, the American Hospital Association, the 
American Medical Association, and the Council of Medical Specialty Societies. Each of these organizations appoints four representatives to the American Council for Graduate Medical Education, whose voting membership also includes two public representatives and a resident.

The American Council for Graduate Medical Education is a public sector organisation that has no attachments to the federal or state governments, although accredited residency programmes receive funding from the federal government through the national health insurance programme, known as Medicare. Hospitals and other training sites are reimbursed through Medicare for a significant percentage of their costs to train residents. The government, however, does not participate in defining the content of the training or how it should be conducted. The participating professional medical groups have been virtually unanimous in their desire to maintain the responsibility for oversight as a private sector function.

\section{Residency Review Committees}

The American Council for Graduate Medical Education delegates the authority to review and accredit thevarious residency programmes to Residency Review Committees, each recognised specialty having its own committee. Each Residency Review Committee has as its sponsors the respective specialty board and the American Medical Association. In most cases, a specialty society is the third sponsor. The Residency Review Committee for surgery, for example, is sponsored by the American Medical Association, the American Board of Surgery, and the American College of Surgeons. The Residency Review Committees are composed of between six and 15 physicians who are appointed for a maximum term of six years by the sponsors. Many of the committee members are themselves past or present directors of residency programmes, or are at least associated with a training programme, and many have achieved national recognition within their specialty. The members serve in a voluntary capacity, with reimbursement for expenses, and appointment to one of these committees is considered prestigious.

\section{Programmes and institutions}

There are approximately 4000 accredited programmes in the 26 primary specialties. ${ }^{\star}$ The American Council for Graduate Medical Education accredits the individual programmes, not the institutions in which they are conducted. The hospitals and other training sites are required to have their own accreditation or licensure through other organisations, such as the Joint Commission for the Accreditation of Health Care Organizations. The American Council for Graduate Medical Education does, however, conduct regular reviews of the sponsoring institutions to verify that appropriate educational policies are in place and that the programmes are receiving adequate support.

\section{Programme requirements}

Each specialty describes its essential training components in its written requirements. These are periodically revised to ensure consistency with current practice and they must be reviewed and approved by the American Council for Graduate Medical Education at least every five years. The requirements for all of the specialties are published annually in a single volume, which also includes the listing of the currently accredited programmes.

The requirements mandate that the residencies be structured educational programmes, which must develop specific goals and objectives and present evidence of an organised plan for assigning progressive clinical responsibility to residents. Unlike the preceptorship model, a programme is expected to provide all residents with the same or comparable experiences, including clinical rotations and formal didactics. In its evaluation of residencies, the Residency Review Committees judges whether the programme demonstrates substantial compliance with these requirements.

The training requirements cover all aspects of the programme, some of which are common to all specialties: the necessity for an adequate volume and variety of patients; a sufficient number of qualified faculty who participate actively in supervising and teaching the residents; assurance of graduated clinical responsibility for residents; an organised didactic programme of conferences and formal teaching sessions; evidence of on-going faculty research and publication; a procedure for regular evaluation and counselling of the residents; and demonstration of support from the sponsoring institution for facilities, space and equipment. Each Residency Review Committees further defines 


\section{Primary specialties with} subspecialties

Allergy and immunology:

clinical and laboratory immunology

Anaesthesiology:

critical care medicine

pain management

Dermatology:

dermatopathology

Emergency medicine:

sports medicine

Family practice:

geriatric medicine

sports medicine

Internal medicine:

cardiovascular disease

clinical cardiac electrophysiology

critical care medicine

endocrinology, diabetes, and metabolism

gastroenterology

geriatric medicine

haematology

haematology/oncology

infectious disease

nephrology

oncology

pulmonary disease

pulmonary disease and critical care medicine

rheumatology

sports medicine

Neurological surgery:

pediatric neurological surgery

Neurology:

child neurology

clinical neurophysiology

Orthopaedic surgery:

adult reconstructive orthopaedics

foot and ankle orthopaedics

hand surgery

musculoskeletal oncology

orthopaedic sports medicine

orthopaedic surgery of the spine

orthopaedic trauma

paediatric orthopaedics

Pathology:

blood banking/transfusion medicine

chemical pathology

cytopathology

dermatopathology

forensic pathology

haematology

immunopathology

medical microbiology

neuropathology

paediatric pathology

Paediatrics:

neonatal-perinatal medicine

paediatric cardiology

paediatric critical care medicine

paediatric endocrinology

paediatric gastroenterology

paediatric haematology/oncology

paediatric nephrology

paediatric pulmonology

paediatric sports medicine

Plastic surgery:

hand surgery

Psychiatry:

addiction psychiatry

child and adolescent psychiatry

geriatric psychiatry

Radiology - diagnostic:

neuroradiology

nuclear radiology

paediatric radiology

vascular/interventional radiology

Surgery - general:

surgical critical care

hand surgery

paediatric surgery

vascular surgery

Urology:

paediatric urology these programme requirements as appropriate to its discipline. In addition, all committees assess whether the programme, in assigning clinical responsibilities to the resident, maintains an acceptable balance between education and service obligations. The Residency Review Committees also monitor resident workload and duty hours. For example, the American Council for Graduate Medical Education has mandated that residents must be given at least one day out of seven free of routine responsibilities and may be on call in the hospital no more than every third night. Some specialties have incorporated into their requirements a maximum number of hours that may be worked by a resident in a week or on a shift. Another dimension of a programme's quality that may be considered by the Residency Review Committees is the success rate of a programme's graduates on the certifying examinations of the specialty board.

Unlike the Canadian system, there is no requirement in the US for residency training to be associated with a medical school. Those institutions sponsoring the largest number of programmes, however, are generally the major medical centres and these usually include a medical school. There may be as many as 65 accredited primary specialty and subspecialty programmes within such an institution.

In most specialties there is a minimum requirement for the presence of at least one other specialty in the same institution. For example, an institution sponsoring a diagnostic radiology programme must also have accredited residency programmes in internal medicine, general surgery and pathology. Orthopaedic surgery requires the presence of both internal medicine and general surgery. Under these circumstances there can be a domino effect if one of the residency programmes loses its accreditation. It is also possible for a community hospital to offer a single programme, for example, in family practice.

\section{Survey/review cycle}

An accredited programme is reviewed every one to five years. The cycle is determined by the Residency Review Committee and depends on a judgment of whether problems exist in the programme and, if they do, how severe they are. In preparation for the survey, a detailed form is completed that requires updated information on all aspects of the training programme. The regular monitoring and review of the programmes involves a site visit. The American Council for Graduate Medical Education employs a cadre of surveyors who visit the programmes to interview the residents, faculty, hospital administrator, and perhaps the medical school dean, to determine the accuracy of the programme's self description. The surveyor's report, together with the forms completed by the programme, are then reviewed at a meeting of the Residency Review Committee.

\section{Limitations of Residency Review Committee responsibilities}

The Residency Review Committees are limited in their mission to the establishment of education standards and to the evaluation of programmes with respect to the standards. They are prohibited from in any way controlling or attempting to manipulate physician supply within the specialties. While the American Council for Graduate Medical Education functions without government control, it is nevertheless subject to scrutiny by an agency of the government, the Federal Trade Commission, an independent agency that was established by Congress in 1914 to have judicial oversight for a wide variety of business circumstances involving competition and restraint of trade. A nonprofit medical association such as the American Council for Graduate Medical Education may not, as part of its activities, control access to any area of medical practice. There has recently been considerable public debate in the US about the country's physician manpower needs, in terms of total supply, and distribution by specialty and geographic location. Despite fairly widespread agreement that more generalists and fewer subspecialists might be desirable, there is no national physician manpower policy. Although the American Council for Graduate Medical Education might be in a position to participate in a redistribution of the medical specialties, it may not do so because it is not exempt from any of the Federal Trade Commission's trade regulations.

\section{Length of training}

The length of residency training may be from two to six years, depending on the specialty. Family practice, internal medicine and anesthesiology are examples of three-year programmes. Diagnostic radiology and obstetrics and gynaecology 
require four-year programmes, and general surgery five years. Certain specialties have a prerequisite or requirement for inclusion of training in other specialties. For specialties involving minimal or focused patient contact, such as radiology and nuclear medicine, a preliminary year of general clinical training is required. Allergy and immunology, which is one specialty, requires prior completion of an internal medicine or pediatrics residency. The surgical specialties all require some additional training. For example, neurological surgery is a five-year programme that requires an additional year of basic clinical training in another accredited programme, such as internal medicine. For thoracic surgery, which is a two-year programme, the prerequisite is prior completion of a four-year general surgery programme.

\section{Subspecialties}

It has become increasingly common for physicians to pursue additional training after completing a residency programme in a primary specialty. In the last five to 10 years, there has been a rapid proliferation of new subspecialty areas. As of 1 January 1995, the American Council for Graduate Medical Education had approved 65 subspecialty areas for accreditation and there are now over 3000 of these programmes, which represents over $40 \%$ of all accredited programmes. Sixteen of the 26 primary specialties have recognised subspecialty areas. Internal medicine includes 15 areas of subspecialisation, such as infectious disease, haematology, electrocardiac physiology, and cardiovascular disease. Under paediatrics, there are nine subspecialty areas, among them paediatric haematology/oncology, nephrology, endocrinology and sports medicine.

In some cases, a subspecialty represents accommodation between two or more primary specialties to offer training and recognition in an area where there is overlap. An examples of this is critical care, which may be conducted by anaesthesiology, internal medicine, paediatrics or surgery. In one large institution, there may be several accredited critical care programmes, each conducted in its own intensive care units, or there may be a combined programme in which several departments collaborate. Geriatric medicine is another example of collaboration, in this case between family practice and internal medicine.

The duration of training in subspecialty programmes ranges from one to three years, and candidates for the programmes must have already completed a residency in the core specialty. Education and certification in a subspecialty area may be important to a physician who is preparing for an academic career in teaching or research, or who plans to restrict her or his practice to a very defined area of medicine. Certainly questions have been raised about the advisability of continuing to approve additional subspecialty areas for certification and accreditation. Fragmentation of the primary specialties, rising costs to the healthcare delivery system, and public confusion about such fine distinctions in medical practices are all subjects of ongoing debate. It appears at least for the present, however, that subspecialisation is a trend that will not easily reverse itself.

\section{Conclusion}

In summary, the accreditation of graduate medical education in the US is accomplished within a cohesive and centralised system that monitors the quality of residency training in all $\mathbf{5 0}$ states and Puerto Rico. Over the last three to four decades, the system has become increasingly focused on the importance of structured educational programmes that will provide comparable training to residents in each specialty in programmes throughout the country. The American Council for Graduate Medical Education, which through its Residency Review Committees, accredits the programmes, and the American Board of Medical Specialties, which approves the specialties and certifies graduates of the accredited programmes, are public sector organisations that complement and cooperate with each other in this endeavour.

American Board of Medical Specialties, Annual Report and Reference Handbook, 1994, pp 42-3.
Graduate Medical Education Directory, 1995/6, 